\title{
ADDENDUM
}

\section{Chemical inhibitor of nonapoptotic cell death with therapeutic potential for ischemic brain injury}

Alexei Degterev, Zhihong Huang, Michael Boyce, Yaqiao Li, Prakash Jagtap, Noboru Mizushima, Gregory D Cuny, Timothy J Mitchison, Michael A Moskowitz \& Junying Yuan

Nat. Chem. Biol. 1, 112-119 (2005); published online 29 May 2005; corrected after print 31 January 2013

In this Article ${ }^{1}$, we described a small-molecule inhibitor of necroptosis, termed Necrostatin-1 (Nec-1). Since the original publication, additional data regarding the properties of $\mathrm{Nec}-1$ have been reported, including off-target activity and metabolic stability in mice, that are important in designing in vitro and, especially, in vivo experiments with $\mathrm{Nec}-1$.

Teng et al. ${ }^{2}$ reported an optimized derivative of Nec-1, termed 7-Cl-O-Nec-1 (66 in ref. 2), that was used in ref. 1 to demonstrate the protection in an ischemic brain injury model. This molecule showed higher activity in inhibiting necroptosis in Jurkat cells than Nec-1 $\left(\mathrm{EC}_{50}=210 \mathrm{nM}\right.$ versus $\left.\mathrm{EC}_{50}=490 \mathrm{nM}\right)$, no nonspecific cytotoxicity at high concentrations $(\sim 100 \mu \mathrm{M})$ and reasonable pharmacokinetic characteristics following intravenous administration in mice. Degterev et al. ${ }^{3}$ subsequently reported that Nec- 1 shows limited metabolic stability, which is substantially improved with 7-Cl-O-Nec-1. Takahashi et al. ${ }^{4}$ also reported that Nec-1 showed paradoxical toxicity at lower, but not higher, doses in a mouse model of systemic inflammatory stress syndrome (SIRS). No such toxicity was observed with 7-Cl-O-Nec-1. Thus, for in-cell and in vivo experiments, we recommend the use of 7-Cl-O-Nec-1.

Muller et al. ${ }^{5}$ reported that Nec-1, also known by its chemical name of methylthiohydantoin-tryptophan, is a micromolar inhibitor of indolamine 2,3-deoxygenase (IDO) with $\mathrm{EC}_{50}=11.4 \mu \mathrm{M}$ in a cell-based assay. Thus, given the $\sim 20$-fold higher activity of Nec-1 in a necroptotic assay, the use of lower concentrations of this molecule could be helpful in distinguishing between inhibition of necroptosis and IDOrelated processes. Another known inhibitor of IDO, 1-methyl-DL-tryptophan, lacks activity against necroptosis as reported by both Degterev et al. ${ }^{3}$ and Takahashi et al. ${ }^{4}$ Notably, both reports show that optimized 7-Cl-O-Nec-1 lacks activity against IDO. Overall, potential nonspecific toxicity, inhibition of IDO and limited stability of $\mathrm{Nec}-1$ should be taken into account when the molecule is used in vivo, whereas 7-Cl-ONec-1 lacks these liabilities and thus represents a superior choice for in vivo studies.

\section{References}

1. Degterev et al. Chemical inhibitor of nonapoptotic cell death with therapeutic potential for ischemic brain injury. Nat. Chem. Biol. 1, 112-119 (2005).

2. Teng et al. Structure-activity relationship study of novel necroptosis inhibitors. Bioorg. Med. Chem. Lett. 15, 5039-5044 (2005).

3. Degterev, A., Maki, J.L. \& Yuan, J. Activity and specificity of necrostatin-1, small-molecule inhibitor of RIP1 kinase. Cell Death Differ. 20, 366 (2012)

4. Takahashi, N. et al. Necrostatin-1 analogues: critical issues on the specificity, activity and in vivo use in experimental disease models. Cell Death Dis. 3, e437 (2012)

5. Muller et al. Inhibition of indoleamine 2,3-dioxygenase, an immunoregulatory target of the cancer suppression gene Bin1, potentiates cancer chemotherapy. Nat. Med. 11, 312-319 (2005)

\section{CORRIGENDUM}

\section{Conformational stabilization of ubiquitin yields potent and selective inhibitors of USP7}

Yingnan Zhang, Lijuan Zhou, Lionel Rouge, Aaron H Phillips, Cynthia Lam, Peter Liu, Wendy Sandoval, Elizabeth Helgason, Jeremy M Murray, Ingrid E Wertz \& Jacob E Corn

Nat. Chem. Biol. 9, 51-58 (2013); published online 25 November 2012; corrected after print 29 January 2013

In the version of this article initially published, the authors neglected to acknowledge an important collaborator and to include a citation of their related work. The error has been corrected in the HTML and PDF versions of the article. 